\title{
Diffraction and scattering studies using high brightness Excillum MetalJet X-ray sources
}

\author{
A Adibhatla ${ }^{1}$, J Hallstedt ${ }^{2}$ \\ ${ }^{1}$ Excillum Inc, ${ }^{2}$ Excillum Inc \\ anasuya.adibhatla@excillum.com
}

X-ray scattering techniques such as SAXS, BIO-SAXS, non-ambient SAXS and GISAXS rely heavily on the X-ray source brightness for resolution and exposure time. Single crystal and powder diffraction experiments also need the brightness and flux to measure time-based phase transitions. Traditional solid or rotating anode x-ray tubes are typically limited in brightness by when the e-beam power density melts the anode. The liquid-metal-jet technology has overcome this limitation by using an anode that is already in the molten state. With bright compact sources, time resolved studies could be achieved even in the home laboratory. We report brightness of $6.5 \times 1010$ photons $/(\mathrm{s} \cdot \mathrm{mm} 2 \cdot \mathrm{mrad} 2 \cdot \operatorname{line})$ over a spot size of 10 $\mu \mathrm{m}$ FWHM.

Over the last years, the liquid-metal-jet technology has developed from prototypes into fully operational and stable X-ray tubes running in more than 100 labs over the world. Multiple users and system manufacturers have been now routinely using the metal-jet anode $\mathrm{x}$-ray source in high-end diffraction and scattering set-ups. With the high brightness from the liquid-metal-jet x-ray source, novel techniques that was only possible at synchrotron before can now also be used in the home lab. This presentation will review the current status of the metal-jet technology specifically in terms of stability, lifetime, flux and optics. It will furthermore refer to some recent powder diffraction and scattering data collected by our users. 